\title{
Determinants of Mortality in Newborn with Sepsis Condition Using Binary Logistic Model at the Tamale Teaching Hospital
}

\author{
Abdul Latif Alhassan'1, M. Sulemana², Mutawakilu Yahaya ${ }^{3}$ \\ ${ }^{1}$ Department of Mathematics and Computer Studies, Tamale College of Education, Tamale, Ghana \\ ${ }^{2}$ Department of Mathematics, University for Development Studies, Navrongo, Ghana \\ ${ }^{3}$ Department of Mathematics and Computer studies, Tamale College of Education, Tamale, Ghana \\ Email: lajoe.lajoe77@gmail.com, wunichee@yahoo.com, ymutawakilu@tace.edu.gh
}

How to cite this paper: Alhassan, A.L., Sulemana, M. and Yahaya, M. (2019) Determinants of Mortality in Newborn with Sepsis Condition Using Binary Logistic Model at the Tamale Teaching Hospital. Open Access Library Journal, 6: e5409. https://doi.org/10.4236/oalib.1105409

Received: April 18, 2019

Accepted: May 27, 2019

Published: May 30, 2019

Copyright $\odot 2019$ by author(s) and Open Access Library Inc.

This work is licensed under the Creative Commons Attribution International License (CC BY 4.0).

http://creativecommons.org/licenses/by/4.0/

\begin{abstract}
Newborn with Sepsis condition has become a major cause of deaths in newborn babies in the world especially in developing countries. This study considered a total of 1019 cases diagnosed with sepsis at the Tamale Teaching Hospital covering the period January 2010 to June 2015. The data was modeled using the Binary logistic regression model to ascertain the variables that contributed to mortality of newborn with sepsis condition at the Tamale Teaching Hospital. Preliminary analysis revealed that pregnant women who mostly failed to attend the antenatal are most affected. Further analysis shows cost of treatment and location of patients as significant contributing variables that contributed to mortality of newborn with sepsis condition at the facility. As a result, it was recommended that facilities must be improved at the districts health centers to cut traveling time of pregnant women to the bigger health centers as well as checking the rising cost of treatment so as to minimize or eradicate newborn with sepsis condition.
\end{abstract}

Subject Areas
Pediatrics
Keywords
Sepsis, Antibiotics, Mortality, Neonates, Logistic, Regression

\section{Introduction}

An acceptable definition of newborn sepsis is lacking according to reports on the expert meeting on neonatal and pediatric sepsis of EMA (2010). It can be de- 
fined by the presence of at least two clinical symptoms in the presence of suspected baby [1]. Newborn sepsis is a severe infection in an infant less than 28 days old. The infection is mostly found in the blood of the infected baby, but can also affect any part of the body system. Newborn sepsis is mostly caused by bacteria. Some other germs can also cause it. A fetus may become infected before birth if the amniotic fluid is infected. During delivery, the newborn may be exposed to an infection in the birth canal. Once born, a baby may be exposed to infections in the hospital. Babies that are mostly at risk, included babies born too early (babies born before thirty-seven weeks) and mothers who do not attend antenatal clinic. Also when the mother's amniotic sac is ruptured for more than 18 hours before birth, it could also lead to sepsis [2]. The incidence of sepsis is the highest in neonates and children, yet the global burden of sepsis in these age groups has not been assessed. They reviewed available evidence from observational epidemiological studies to estimate the global burden and mortality of sepsis in neonates and children. We did a systematic review and meta-analysis of studies reporting population-based sepsis incidence in neonates and children, published between 1979 and 2016. Their search yielded 1270 studies, 23 of which met the inclusion criteria; 16 were from high-income countries and seven from middle-income countries. 15 studies from 12 countries reported complete data and were included in the meta-analysis. We found an aggregate estimate of 48 (95\% CI 27 - 86) sepsis cases and 22 (14 - 33) severe sepsis cases in children per 100,000 person-years. Mortality ranged from $1 \%$ to $5 \%$ for sepsis and $9 \%$ to $20 \%$ for severe sepsis. The population-level estimate for neonatal sepsis was 2202 (95\% CI 1099 - 4360) per 100,000 live births, with mortality between $11 \%$ and $19 \%$. Extrapolating these figures on a global scale, we estimate an incidence of three million cases of sepsis in neonates and one million two thousand million cases in children. Although these results confirm that sepsis is a common and frequently fatal condition affecting neonates and children globally, few population-based data are available from low-income settings and the lack of standardization of diagnostic criteria and definition of sepsis in the reviewed studies are obstacles to the accurate estimation of global burden. Robust epidemiological monitoring to define global sepsis incidence and mortality in children is urgently needed. According to [3], the proportion of child deaths that occur in the neonatal period (38\% in 2000) is increasing, and the Millennium Development Goal for child survival cannot be met without substantial reductions in neonatal mortality. Every year an estimated four million babies die in the first 4 weeks of life (the neonatal period). A similar number is still born and five hundred thousand mothers die from pregnancy-related causes. Three-quarters of neonatal deaths happen in the first week. The highest risk of death is on the first day of life. Almost all (99\%) neonatal deaths arise in low-income and middle-income countries, yet most epidemiological and other research focuses on the $1 \%$ of deaths in rich countries. The highest numbers of neonatal deaths are in south-central Asian countries and the highest rates are generally in sub-Saharan Africa. The countries in these regions (with some exceptions) have made little 
progress in reducing such deaths in the past 10 - 15 years. Globally, the main direct causes of neonatal death are estimated to be preterm birth (28\%), severe infections (26\%), and asphyxia (23\%). Neonatal tetanus accounts for a smaller proportion of deaths (7\%), but is easily preventable. Low birth weight is an important indirect cause of death. Maternal complications in labor carry a high risk of neonatal death, and poverty is strongly associated with an increased risk. Preventing deaths in newborn babies has not been a focus of child survival or safe motherhood programs. While we neglect these challenges, 450 newborn children die every hour, mainly from preventable causes, which is unconscionable in the 21 st century [4]. Component of all child mortality, now constituting about $40 \%$ of child deaths. As attention As infant and child mortality declines in many developing countries, neonatal mortality becomes the dominant is geared towards the problem, it has become obvious that, in the high mortality countries, neonatal mortality rates are being systematically underestimated, especially in the low income and underdeveloped countries. Data on the causes of neonatal deaths in the community are inadequate, as mortality occurs outside the health centers [5]. Neonatal sepsis causes an estimated 750,000 annual deaths worldwide with mortality highest in sub-Saharan Africa (SSA). The third Sustainable Development Goal for child health, which aims to end preventable deaths of newborns and children less than 5 years of age by 2030 , cannot be met without substantial reduction of infection-specific neonatal mortality in the developing world. Neonatal infections are estimated to account for $26 \%$ of annual neonatal deaths, with mortality rates highest in sub-Saharan Africa (SSA). Reliable and comprehensive estimates of the incidence and aetiology surrounding neonatal sepsis in SSA remain incompletely available [6].

In April, 2018, a paper was written on the topic, "Predictors of Neonatal Deaths in Ashanti Region of Ghana" Neonatal mortality continues to be a public health problem, especially in sub-Saharan Africa. Their study was conducted to assess the maternal, neonatal, and health system related factors that influence neonatal deaths in the Ashanti Region, Ghana. Out of the 222 mothers, there were 115 (51.8\%) whose babies did not survive. Majority, 53.9\%, of babies died within 1 - 4 days, 31.3\% within 5 - 14 days, and $14.8 \%$ within 15 - 28 days. The study found out that, asphyxia, low birth weight, congenital anomalies, infections, and respiratory distress syndrome. Neonatal deaths were influenced neonatal factors (birth weight, gestational period, sex of baby, and Apgar score), and health related factors (health staff attitude, supervision of delivery, and hours spent at labor ward). Their research concluded that, there was a high level of neonatal deaths in the Ashanti Region of Ghana. According to [7] Sepsis in newborns can be hard to identify. Some of the symptoms in newborn babies may include but not limited to the following; Signs of distress during labor or delivery, such as a rapid heart rate, Changes in temperature (usually fever), breathing problems, such as very fast breathing, Digestive problems like poor appetite or an enlarged liver, Nervous system [8].

An article on the topic, "Risk Factors Associated with Neonatal Sepsis" ac- 
cording to the study, neonatal sepsis accounts for an estimated $26 \%$ of under-five deaths, with sub-Saharan Africa having the highest mortality rates. It remains a notable hindrance to the progress in the decline of cause-specific mortality rates especially in sub-Saharan Africa. This study aimed at examining the risk factors of neonatal sepsis at the Trauma and Specialist Hospital, Winneba. The study found both maternal and neonatal factors to have a strong association with the risk of developing neonatal sepsis [9].

According to the [7], the signs of sepsis are similar to many other health conditions. A baby may need tests. These may include: Blood culture to check for bacteria in the blood but results of such test take a few days. Mostly when sepsis is suspected, treatment start right away in order not to allow situation to worsen. The blood culture and other ways are used to diagnose sepsis. (Urine culture to check for bacteria in the urinary system and other cultures to check for bacteria in other places). A quick X-ray on the chest could also be used check for a lung infection. Early diagnosis and treatment is the best way to stop sepsis. If a baby health care provider suspects sepsis, the baby is expected to be given antibiotics right away, even before test results are available. Once the provider has the test results, which may confirm sepsis or otherwise, may continue or change treatment. A newborn with sepsis may be very ill. He or she will need to stay in the newborn intensive care unit (NICU). In the NICU the baby will be watched very closely whiles antibiotics such as ampicillin, gentamicin, cefotaxime, vancomycin, metronidazole, erythromycin, and piperacillin are given. Other supplementary support such as oxygen, nutrition may be provider if necessary. Some of the complications of Sepsis is life-threatening for newborns. It often affects more than one system at the same time. Although newborn sepsis can't be completely prevented, you can lower the risk. Regular prenatal care can detect and treat many problems that put a newborn at risk for sepsis. Hospitals and other facilities have practices in place to prevent the spread of infection. These include: Frequent hand-washing, Limiting procedures that involve cutting or putting instruments into the body etc. There has been very scanty scientific study on the issue of newborn sepsis in the northern Ghana. The issue of key variables which influence the mortality of newborn sepsis at the hospital is given little or no attention. It is as a result of this that, this study is intended to look at the variables that determines the mortality of newborn sepsis in the only referral teaching hospital in the northern region [10].

\section{Materials and Methods}

This comprises of the data management, which included the study facility, the region of study, the population of the area the coding of variables and how the data was extracted.

\subsection{Data Management and Sample Size}

The data used in this study was a secondary data from Tamale Teaching Hospit- 
al. Tamale Teaching Hospital is located in the capital of Northern Region-Ghana. Ghana is located in West Africa. It has a total population of over twenty four million people $(24,000,000)$ The sample size was one thousand and nineteen (1019). It covers the period from January 2010 to June 2015. This data was extracted from among other ailments from the District Health Information Management System (DHIMS). Tamale has a total population of 2,468,557 people (2010 population census) [11]. A design matrix was formed after the extraction of the data, and the variables captured included the following, gender (sex), outcome of admission, location of patient (distance), insurance of patient, cost of treatment and duration of stay. These variables were all coded as follows: gender (female $=\mathrm{f}$, male $=\mathrm{m}$ ), outcome of admission was categorized into two, either patient was discharged or died (discharged $=\mathrm{d}$, death $=\mathrm{t}$ ), location of patient was considered in terms of distance of the patient from his/her residence to the hospital. This was classified into two categories and coded as (less than 10 kilometers $=1$, greater or equal to 10 kilometers $=2$ ), insurance status of the patient was also categorized into two and coded as yes $=$ insured, no $=$ not insured.

\subsection{Concept of Binary Logistic Models and Model Building}

Both discriminant analysis and logistic regression can be used to determine the categorical probability of an event occurring given a selected number of continuous and categorical variables. However, discriminant analysis requires that the distribution of independent variables in the model follow a normal distribution [12]. Logistic regression is the recommended statistical procedure when dichotomous variables are used (Sharma, 1996). Logistic regression also does not require independent variables to be linearly related, nor does it require equal variance within each group, which also makes it a less stringent procedure for statistical analysis (Starkweather \& Moske, 2011). (As a result, logistic regression was used to determine the mortality of newborn sepsis [12]. Logistic Regression analysis uses maximum likelihood estimation to predict group membership. However, to interpret the results of the prediction of group membership with precision and accuracy. One assumption of logistic regression is that the continuous predictors of the model are linear with the logit of the dependent variables. Hosmer and Lemeshow (2000) recommends using the Box-Tidwell approach to check for the linearity of the logit (as cited in Tabachnick \& Fidell, 2013). Using this method, a Logistic regression model was created regressing the dependent variable on each of the continuous predictors, and their interaction terms, which consists of the continuous Predictors and its natural log. If at least one interaction is significant then the assumption is violated and a transformation of the variable was considered (Tabachnick \& Fidell, 2013).

A limitation of logistic regression is that it is sensitive to variables that have very high correlations with each other. Variables that are highly collinear often produce very large standard errors and inflated regression estimates (Tabachnick \& Fidell, 2013). Therefore, the collinearity between the independent va- 
riables in the model had to be observed. A standard procedure that allows for this is the calculation of tolerance for each variable. The tolerance statistic is the calculation of the variance of each of the independent variables in the model not explained by all of the other independent variables in the model. A higher tolerance value suggests low levels of collinearity. Menard (2010) suggests that a tolerance of less than 0.2 is alarming. Although logistic regression software does not typically offer a tolerance function, Menard (2010) suggests that the model be calculated as linear regression to observe the relationship among independent variables [13].

1) Preliminary classification shows from the decision variable in Table 1. That, $111 / 1017=0.109$ given an average percentage of $11 \%$ for discharged whiles $89 \%$ died. By these classifications we can correctly conclude that, $89 \%$ couldn't survive the sepsis whiles the remaining $11 \%$ survived. We could see that, the overall success rate for using this model is $89 \%$.

Table 2 Shows the test for Hosmer and Lemeshow test which is intended to verified if the model is adequate as well as showing whether the data is properly classified. With an hypothesis below, we can conclude that, the model is adequate for the data comparing the a significant value of 0.05 to the Hosmer and Lemeshow value of 0.007 . We therefore reject the null hypothesis that, "the model is not adequate for the data" in favor of the alternative hypothesis since $0.007<0.05$.

2) The mathematical concept of logistic regression is to express the relationship between outcome variable and predictor variables (independent variables) in terms of logit: the natural logarithm of odds. Considering a case where $Y$ is a dichotomous outcome variable categorized as " 1 " and " 0 " and $X$ is a continuous predictor variable. Logistic regression facilitates a situation by logit transforming on the outcome variable $Y$. The logistic regression model can be written as:

$$
\operatorname{logit}(y)=\ln \frac{\mu}{1-\mu}=\beta_{0}+\beta_{1} X
$$

here $\mu$ is the probability of occurring the outcome $Y$ and $\left(\frac{\mu}{1-\mu}\right)$ is the odds of success; the ratio of the probability of occurring the outcome $Y$ and the probability of not occurring the outcome $Y . \beta 0$ and $\beta 1$ are called intercept and slope (regression coefficient) respectively. By taking antilog on both sides of Equation (1) we can estimate the probability of the occurrence of outcome $Y$ for a given value of predictor

$$
X: \mu=p\left(\frac{Y}{X}=x\right)=\frac{\mathrm{e}^{\beta_{0}+\beta_{1 X}}}{1+\mathrm{e}^{\beta_{0}+\beta_{1 X}}}
$$

We can extend the logistic model in Equation (2) for more than one predictor as in the case of this study

$$
\log i t(Y)=\ln \frac{\mu}{1-\mu}=\beta 0+\beta 1 X 1+\beta 2 X 2+\beta 3 X 3+\beta 4 X 4+\beta 5 X 5
$$


Table 1. Classification Table.

\begin{tabular}{cccc}
\hline & \multicolumn{3}{c}{ Predicted } \\
\cline { 2 - 3 } Observed & \multicolumn{2}{c}{ Outcome of Treatment } & Percentage Correct \\
\cline { 2 - 3 } & Discharge & Died & 0.0 \\
Discharge & 0 & 111 & 100.0 \\
Died & 0 & 906 & 89.1 \\
Total & & & \\
\hline
\end{tabular}

Table 2. Hosmer and Lemeshow Test.

\begin{tabular}{cccc}
\hline Step & Chi-square & Df & Sig. \\
\hline 1 & 20.979 & 8 & 0.007
\end{tabular}

Sig. $(p<0.05)$, Ho: "The model is not adequate for the data", H1: The model is adequate for the data".

Equation (3) is the specific form of logistic regression model for five numbers of predictors. Regression parameter $\beta$ s can be estimated by either maximum likelihood (ML) method or weighted least square method. The value of regression coefficients $\beta 1 \ldots \beta 5$ indicate the relationship between $X$ s and logit of $Y$. Coefficient value bigger than 0 indicates an increase in logit of $Y$ with an increase in $X$ and coefficient value smaller than 0 indicates a decrease in logit of $Y$ with an increase in $X$. When the coefficient value is 0 , it indicates there is no linear relationship among logit of $Y$ and predictors $X$.

3) The predictive variables which consist of dataset of gender (categorical variable), the cost in Ghana cedis (continuous variable), the location in kilometers (categorical variable), insurance (categorical variable) and duration (continuous variable) depending on the outcome of treatment of a dichotomous variable (Discharged and Died), the model can be fitted as:

$$
\begin{aligned}
& \text { Logit }(y=1) \\
& =\beta_{0}+\beta_{1} \text { Gender }+\beta_{2} \text { Cost }+\beta_{3} \text { Location }+\beta_{4} \text { Insurance }+\beta_{5} \text { Duration }
\end{aligned}
$$

The null hypothesis of the overall model states that all regression coefficients $(\beta 0, \beta 1, \beta 2, \beta 3, \beta 4, \beta 5)$ are zero. Rejection of this null hypothesis will imply that at least one regression coefficient is non-zero meaning the logistic regression Equation in (4) predicts from the results in Table 3 the fitted of the logistic regression model is as:

$$
\begin{aligned}
& \text { logit }(\text { outcome }) \\
& =0.82+0.05 \text { gender }+0.02 \text { cost }+0.5 \text { location }+19.28 \text { insurance }+0.01 \text { duration }
\end{aligned}
$$

All the predictors "gender", "cost", "location", "insurance" and "duration" are positively related to the log of odds of the independent variable (outcome) as seen in Table 3. The $\mathrm{p}$ value $(\mathrm{p}<0.05)$ indicates the significance of each predictor from Table 3 .

It can be observed that, with an odds ratio of 1.049, male newborns are more likely to die of sepsis as compared to their female counterparts. Again, the odds 
Table 3. Variables in the Equation.

\begin{tabular}{ccccccc}
\hline Variables & B & S.E. & Wald & Df & Sig. & Exp(B) \\
\hline Gender & 0.048 & 0.205 & 0.055 & 1 & 0.814 & 1.049 \\
Cost & 0.018 & 0.004 & 15.761 & 1 & 0.000 & 1.018 \\
Location & 0.548 & 0.205 & 7.142 & 1 & 0.008 & 1.730 \\
Insurance & 19.277 & $11,274.163$ & 0.000 & 1 & 0.999 & $235,473,798.287$ \\
Duration & 0.011 & 0.022 & 0.261 & 1 & 0.609 & 1.011 \\
Constant & 0.816 & 0.307 & 7.060 & 1 & 0.008 & 2.261 \\
\hline
\end{tabular}

Sig. $(\mathrm{p}<0.05)$.

ratio for location, 1.730, indicates that newborn with sepsis who travels at least $10 \mathrm{~km}$ to access health care have higher chance of being dead as compared to those who resides within less than $10 \mathrm{~km}$ radius of the Tamale Teaching hospital. Meanwhile, as cost of treatment increases there is likelihood of increases in death of newborns with sepsis.

It can generally be seen that, the cost of treatment and the location of the patient were the major contributing variables that, causes mortality among newborns with sepsis at the Tamale Teaching hospital within the period under study and therefore considered to be significant with 0.000 and 0.008 respectively. The other variables gender, insurance and duration of stay of patients contributed but their contributions according to the analysis were not significant since their significance values were greater than the $\mathrm{p}$ value of 0.05 .

\section{Summary}

Neonatal sepsis is an acute infection on newborns which can be categorized into two: early-onset or late-onset. Early onset is mostly common since those infections are contracted through the birth canal of the mother during deliveries which mostly occur within 24 hours. Late-onset occurs after 24 hours contracted within the environment [14].

The Binary Logistic Regression model was utilized for this study. It was realized that the cost of treatment and the location of patients were statistically significant in contributing to the mortality in newborn with sepsis with their $\mathrm{p}$-values less than 0.05 . It can be explained that the value 0.816 is seen to be the log of odds for outcome of treatment (Discharged and Died) status when all the variables in question are zero. It was observed that male newborns are more likely to die of sepsis as compared to their female counterparts. Again, it was indicated that the newborn with sepsis who travels at least $10 \mathrm{~km}$ to access health care have higher chance of being dead as compared to those who stayed within less than $10 \mathrm{~km}$ radius of the Tamale Teaching Hospital. The location of patients when other variables are constant will have a unit increase in kilometers by $54.8 \%$ whiles keeping other variables constant, a unit of time in days will increase the outcome of treatment by $1.1 \%$. 


\section{Consent}

The data used required no permission from the patients since the identities of the patients were not in known and therefore not applicable here.

\section{Ethical Approval}

Not applicable.

\section{Authors' Contributions}

This work was carried out in collaboration between all authors. Author ALA worked on the background, methodology and analysis of the data. Author MS worked on the interpretation and literature review. Author MY worked on discussions and the conclusion. All authors read and agreed on the final manuscript.

\section{Conflicts of Interest}

The authors declare no conflicts of interest regarding the publication of this paper.

\section{References}

[1] Aline, F., Julia, B., Shrey, M., Mike, S., Johannes, N. and Van, D.A. (2016) Antibiotic Use for Sepsis in Neonates and Children, Evidence Update Division of Clinical Pharmacology, Children's National Health System, Washington, DC, USA.

[2] Fleischmann-Struzek, C., Goldfarb, D.M., Schlattmann, P., Schlapbach, L.J., Reinhart, K. and Kissoon, N. (2018) The Global Burden of Paediatric and Neonatal Sepsis: A Systematic Review. The Lancet Respiratory Medicine, 6, 223-230. https://doi.org/10.1016/S2213-2600(18)30063-8

[3] Joy, E.L., Simon, C. and Jelka, Z.M. (2015) Neonatal Survival Steering Lancert Team.

[4] Mulholland, E.K., Fenn, E. and Zaidi, A. (2010) Global Burden of Neonatal. Sepsis. International Journal of Infectious Diseases, 14, e181. https://doi.org/10.1016/j.ijid.2010.02.1887

[5] Ranjeval, L.S., Benjamin, C.W. and Steven, J.S. (2018) Economic Burden on Neonatal Sepsis in Sub Sahara Africa. BMJ Global Health, 3. https://doi.org/10.1136/bmjgh-2017-000347

[6] Asia, J., Herman, L., Wella, A.S., Adellah, S. and Joyce, P. (2016) Prevalence and Factors Associated with Neonatal Sepsis among Neonates in Temeke and Mwananyamala Hospitals in Dares Salaam, Tanzania. Tanzania Journal of Health Research, $18,1-7$.

[7] Health Encyclopedia. (2019)

[8] Gertrude, N.A. and Yvonne, A. (2018) Predictors of Neonatal Deaths in Ashanti Region of Ghana. Advances in Public Health, 2018, Article ID 9020914. https://doi.org/10.1155/2018/9020914

[9] Adatara, P., Agani, A., Solomon, M., Salia, R., Adongo, A., Kennedy, D., Konlan, E., Agyabeng, F., Ethel, A., Esther, A.A. and Irene, G.B. (2019) Risk Factors Associated with Neonatal Sepsis: A Case Study at a Specialist Hospital in Ghana. The Scientific 
World Journal, 2019, Article ID 9369051. https://doi.org/10.1155/2019/9369051

[10] Sanghvi, K.P. andTudehope, D.J. (1996) Neonatal Bacterial Sepsis in a Neonatal Intensive Care Unit: A 5 Year Analysis. Journal of Paediatrics and Child Health, 32, 333-338. https://doi.org/10.1111/j.1440-1754.1996.tb02565.x

[11] Population and Housing Census (2010) Ghana.

[12] Abedin, T., Chowdhury, Z., Afzal, A.R., Yeasmin, F. and Turin, T.C. (2016) Application of Binary Logistic Regression in Clinical Research. Department of Family Medicine, Cumming School of Medicine, University of Calgary, Calgary, Alberta, Canada.

[13] Ralph, A., Franciosi, M.D., Blaise, E. and Favara, M.A. (2017) Single Blood Culture for Confirmation of the Diagnosis of Neonatal Septicemia. American Journal of Clinical Pathology, 57, 215-219. https://doi.org/10.1093/ajcp/57.2.21

[14] Yibeltal, T., Seida, M., Birhanie, M., Mequanint, F., Tsigiereda, D., Amare, B., Ashagre, M. and Habte, B. (2016) Clinical Outcome and Risk Factors of Neonatal Sepsis among Neonates in Felege Hiwot Referral Hospital, Bahir Dar, Amhara Regional State, North West Ethiopia. 